\title{
Fully Automated and Adaptive Detection of Amyloid Plaques in Stained Brain Sections of Alzheimer Transgenic Mice
}

\author{
Abdelmonem Feki ${ }^{1,2}$, Olivier Teboul ${ }^{1,2}$, Albertine Dubois ${ }^{1}$, \\ Bruno Bozon $^{3}$, Alexis Faure ${ }^{3}$, Philippe Hantraye ${ }^{1}$, Marc Dhenain ${ }^{1}$, \\ Benoit Delatour ${ }^{3}$, and Thierry Delzescaux ${ }^{1}$ \\ ${ }^{1}$ MIRCen, URA CEA-CNRS 2210, Orsay, France \\ thierry.delzescaux@cea.fr \\ ${ }^{2}$ Ecole Centrale Paris, Grande Voie des Vignes, Chatenay-Malabry, France \\ ${ }^{3}$ Laboratoire NAMC, CNRS, UMR 8620, Université Paris Sud, Orsay, France
}

\begin{abstract}
Automated detection of amyloid plaques (AP) in post mortem brain sections of patients with Alzheimer disease (AD) or in mouse models of the disease is a major issue to improve quantitative, standardized and accurate assessment of neuropathological lesions as well as of their modulation by treatment. We propose a new segmentation method to automatically detect amyloid plaques in Congo Red stained sections based on adaptive thresholds and a dedicated amyloid plaque/tissue modelling. A set of histological sections focusing on anatomical structures was used to validate the method in comparison to expert segmentation. Original information concerning global amyloid load have been derived from 6 mouse brains which opens new perspectives for the extensive analysis of such a data in 3-D and the possibility to integrate in vivo-post mortem information for diagnosis purposes.
\end{abstract}

\section{Introduction}

Alzheimer disease (AD) is a progressive neurodegenerative disorder that affects a large proportion of the ederly population [1. One of the characteristic of histological sections from the brain of patients who had the disease is the presence of many amyloid plaques. These lesions are extracellular deposits of an amyloid protein. They can be detected after histological processing such as tissue staining by the Congo Red method. Each plaque measures approximately from 20 to 200 $\mu \mathrm{m}$. Transgenic mice presenting amyloid plaques (AP) are widely studied to improve our understanding of the pathophysiology of AD but also to investigate new therapeutics. The automated detection of amyloid plaques on large dataset of histological sections remains mainly manual or semiautomatic and based on basic image processing methods using histogram or color image analysis [2]. More recently, an approach using statistical model based on prior operator expertise has been proposed [3]. Nevertheless, most of these approaches remain tedious and time consuming (manual delineation, threshold adjustement). 
In this paper, we propose a fully automated method to detect amyloid plaques in Congo Red stained sections of transgenic mouse brains. The first step consisted in the separation of the tissues from the background based on an expectation maximization (EM) algorithm followed by the extraction of the seeds corresponding to the plaques using global/local adaptive thresholds. The second step exploited the seed and the corresponding region previously extracted to fit a mathematical model estimating iteratively a plane with uniform variance for the tissues and a gaussian surface for the amyloid plaques. The results were then filtered using an a priori area and shape constraints. The processes were implemented in 2-D and could be extented to series of stacked sections (3-D volume). Several informative parameters such as amyloid load (in the section as well as in the whole brain), plaque number, area, distribution and R, G, B intensities could be robustly and reproducibly assessed with the proposed method.

\section{Materials ans Methods}

\subsection{Biological Materials}

During our studies, image analysis were performed on histological sections from hemibrains of APP/PS1 and APP/PS1KI mouse models of AD. The brains were cut into 40 microns-thick coronal sections on a freezing microtome. Brain sections were stained by the Congo Red method. All sections were then digitized using a Nikon Coolscan 4000 ED scanner as RGB images with a 4000 dpi inplane digitization resolution (pixel size $6.35 \mathrm{e}-3 \times 6.35 \mathrm{e}-3 \mathrm{~mm}^{2}$ ) generating large images $(1300 * 1300$ pixels)(Fig.1a). Image analysis was conducted during two successive experiments. First, the method was tested in selected brain regions (hippocampus and frontal part of the brain) from $5 \mathrm{APP} / \mathrm{PS} 1$ and $5 \mathrm{APP} / \mathrm{PS} 1 \mathrm{KI}$ mice. The APP/PS1 mice displayed large amyloid plaques while plaques were smaller in APP/PS1KI animals (Fig.1c). This experiment was conducted to evaluate the robustness of automatic detection under a variety of histological contexts. In a second analysis, plaque detection was evaluated from histological sections that covered the whole brain of $6 \mathrm{APP} / \mathrm{PS} 1$ animals. This allowed to extend the study to different brain regions. For this experiment, one histological brain section out of twelve was extracted so as to finally keep 12-14 sections for each brain with an associated inter-slices thickness of $0.48 \mathrm{~mm}$.

\subsection{Overall Protocol}

From RGB images, G color component was extracted and corresponding gray scales were inverted (Fig.1b). This channel is predominantly used in histological image processing due to the lower level of noise and its high natural contrast 4 . A binary mask of the brain section was computed with EM procedure [5] which estimated a threshold value under hypothesis that intensities of the background and the tissue can be assimilated to gaussian distributions.

The definition of seeds corresponding roughly to amyloid plaques (AP) was performed in a 2-step scheme. To discriminate plaques from tissue, we assumed 


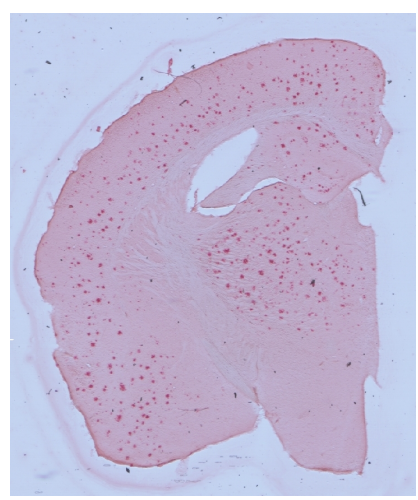

a)

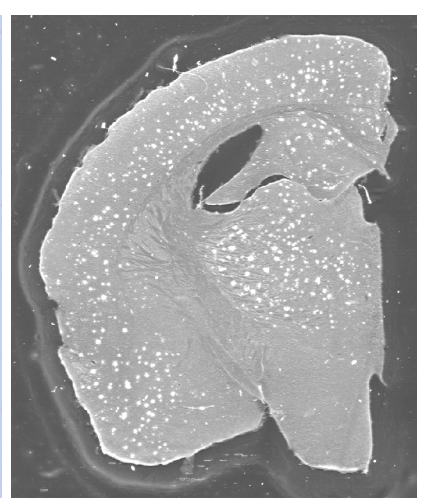

b)

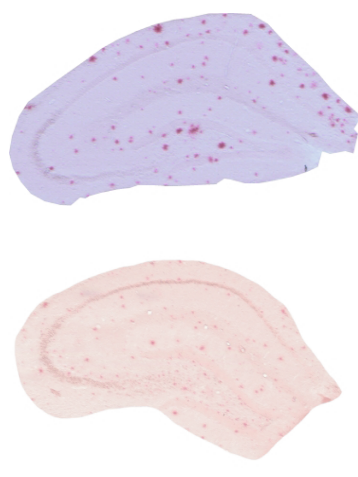

c)

Fig. 1. a) Coronal section stained using Congo Red, b) inverted green color component and c) large/small amyloid plaques respectively in APP/PS1 (top) and APP/PS1KI (bottom) hippocampus

that tissue was the majority class in the section. For a region of interest defined (mask and corresponding image), mean $m$ and standard deviation $\sigma$ values were calculated and pixels presenting a value over $T=m+\sigma$ were automatically outlined and labelled as seeds. To select the optimal contrast between plaques and tissue stainings, this approach was applied twice: 1) on the global masked section to generate a rough estimation of the seeds which was used to create a first generalized Voronoi's partition of the image domain [67] (calculation based on a distance criterion to attribute pixels to the nearest seed) and 2) on each Voronoi's partition (previously obtained in 1) ) to refine both second seeds estimation and consecutively Voronoi's partitions. We proposed a mathematical modelling of AP and brain tissue, and used an interative EM-like approach to separate these to classes.

\subsection{Mathematical Model}

The divide-and-conquer strategy chosen lead to an easier segmentation problem: separating a plaque $(\mathcal{P})$ from the tissue $(\mathcal{T})$. As a consequence, we introduce a model for each class (tissue, AP). The classification algorithm works in two steps: estimate the parameters of the models and classify the pixels until convergence.

Plaque Model. We are looking for an amyloid plaque, which is actually a load of amyloids. As the Congo Red stains these proteins, the intensity of Congo Red is proportional to the density of amyloid. Thus, we consider the intensity of Congo Red in a pixel as a measure of the amyloid load in this pixel's area. Moreover, we suppose that in a plaque, the proteins are distributed according to a spatial gaussian law around the center of the plaque: 


$$
p(X \mid \mathcal{P})=\frac{1}{2 \pi \operatorname{det}\left(\Sigma_{p}\right)^{1 / 2}} \exp \left(-\frac{1}{2}\left(X-\mu_{p}\right)^{T} \Sigma_{p}^{-1}\left(X-\mu_{p}\right)\right)
$$

where $X=(x, y)^{T}$

This meant that the location $X$ of a amyloid protein is a random variable whose probability is $p(X)$. Besides, we assume that the observed intensity $I$ in that location $X$ matches with $I$ observations of that random variable.

However, we don't observe only amyloid protein stains with Congo Red but also the brain tissues which have to be taken into account.

Tissue Model. We assume that the brain intensity follows a gaussian law:

$$
p(I \mid \mathcal{T})=\frac{1}{\sqrt{2 \pi} \sigma_{b}} \exp \left(-\frac{\left(I-\mu_{b}\right)^{2}}{2 \sigma_{b}^{2}}\right)
$$

This meant that the probability of observing an intensity $I$ in the tissue is independant of the location, and is equal to $p(I)$. The main difficulty of this formulation is that the observations don't belong to the same spaces: in the plaque model, the observations are the locations of proteins, and in the tissue model, the observations are the pixels intensities. As a consequence, we use an heuristic to classify the pixels between the two classes, based upon the posterior probabilities of the pixels to belong to these two classes. The algorithm works in two steps: estimate and classify.

Initialization. Let $\tau_{n}$ be the ownership of a pixel $n$ to the plaque: $\tau=1$ if the pixel is in the plaque and 0 otherwise. We intialize $\tau$ to 1 in the vornoi seed, and 0 elsewhere.

Parameters estimation. The estimation of the parameters is based upon the maximum likelihood of the two classes.

$$
\begin{array}{rlrl}
\mu_{b} & =\frac{\sum_{n}\left(1-\tau_{n}\right) I_{n}}{\sum_{n}\left(1-\tau_{n}\right)} & \sigma_{b}^{2} & =\frac{\sum_{n}\left(1-\tau_{n}\right)\left(I_{n}-\mu_{b}\right)^{2}}{\sum_{n}\left(1-\tau_{n}\right)} \\
\mu_{p}=\frac{\sum_{n} \tau_{n} I_{n} X_{n}}{\sum_{n} I_{n} \tau_{n}} & \Sigma_{p} & =\frac{\sum_{n} \tau_{n} I_{n}\left(X_{n}-\mu_{p}\right)\left(X_{n}-\mu_{p}\right)^{T}}{\sum_{n} I_{n} \tau_{n}}
\end{array}
$$

Classification. In order to classify a pixel, we compute its posterior probability (score $S$ ) in the two classes using (112) and the prior probability of each class. We label the pixel by comparing the two scores. For instance, if a pixel have an intensity close to the tissue intensity, the score for the tissue class is high (same intensity) whereas the score for the plaque class is low (because it is far from the plaque center).

$$
\begin{aligned}
S_{p}\left(v_{i}\right) & =\pi p\left(X_{i} \mid \mathcal{P}\right) \\
S_{t}\left(v_{i}\right) & =(1-\pi) p\left(I_{i} \mid \mathcal{T}\right)
\end{aligned}
$$

where $S_{p}\left(v_{i}\right)$ and $S_{t}\left(v_{i}\right)$ are the scores of the voxel $v_{i}$ with respect to both classes, and $\pi$ is the prior probability of the AP class (It is equal to the number of pixels with $\tau=1$ in the previous iteration, divided by the total number of pixels). 


\subsection{Data Analysis}

The amyloid load which was the ratio between the quantity of amyloid plaques and the tissue was the main information to be calculated to characterize ADrelated pathology. Due to the processing on 2-D data, we assumed that the ratio of the surfaces was proportional to the ratio of the volume enabling us to assess amyloid load for both individual sections (validation part) and series of sections (whole brain) according to the principle of Delesse validated in stereology. In addition, shape, position features and intensity of each individual plaque were also computed.

In a first analysis, the proposed methodology was applied to a subset of 20 images focusing on two anatomical structures manually delineated by an expert: the hippocampus $(n=10)$ and the frontal region $(n=10)$. The computation of the amyloid load of reference for each region was performed by a biologist expert using Visilog software and a dedicated image processing protocol (R, G, B color component adjustment, global automated threshold based on entropy criterion and morphometric filtering according to Feret's diameter). A correlation analysis was performed to test the linear relationship between the measurements of the two methods.

The second part of the work consisted in analysing 6 mouse brains (representing 77 sections) in order to derive global parameters and to perform an inter-subject comparison. The AP and tissue areas were automatically calculated for each section and brain accross antero-posterior axis. In the end, visual inspection of the results based on superimposed image of the original section and its segmentation image as well as a volume rendering of the plaques in 3-D were performed.

\section{Results}

Figure 2 describes the sequential results obtained at different step of the AP segmentation in one section (Fig $2 \mathrm{a}$ ). Following the automated extraction of the section performed on the inverted green color component, holes corresponding to the ventricles and vessels can be observed (Fig 2b).

The definition of subregions including a single seed was performed in a 2-steps process: the first one provided a rough estimation of the seeds which generated several large Voronoi's partitions coded with a Rainbow colormap (Fig 2:) while the second step produced an oversegmentation leading to supplementary seeds detected as well as small and numerous Voronoi's partitions (Fig_2d). This information was then treated with the mathematical model proposed in order to filter real AP from artefacts (border, dust, vessels, stria) and to assess quantitative parameters (location, area, intensity). We proposed a pseudo 3D representation of the detected AP as spheres (presenting a size and a color texture relative to their mean radius and facilitating the visualization of the detection on several sections) which were superimposed on the mask of the section (Fig 2 $\mathrm{b}$ ). Masked 


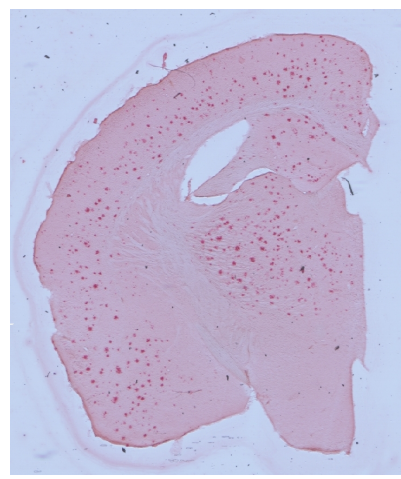

a)

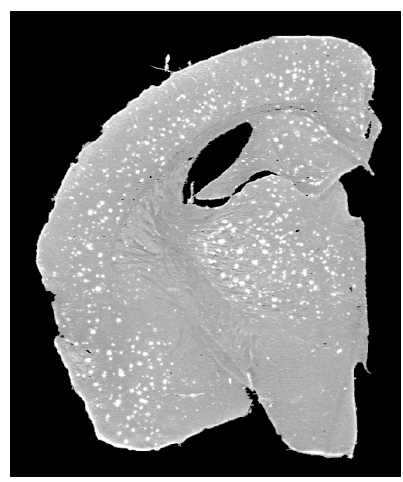

b)

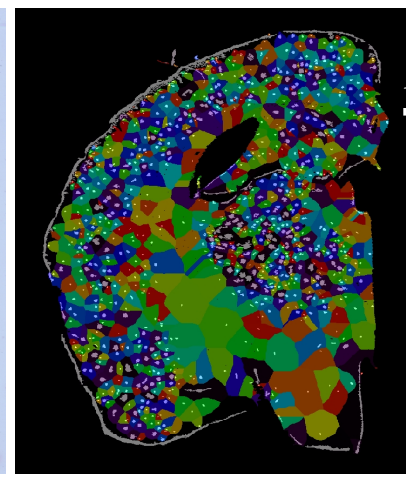

c)

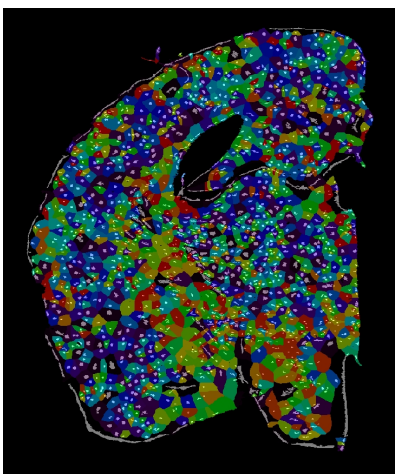

d)

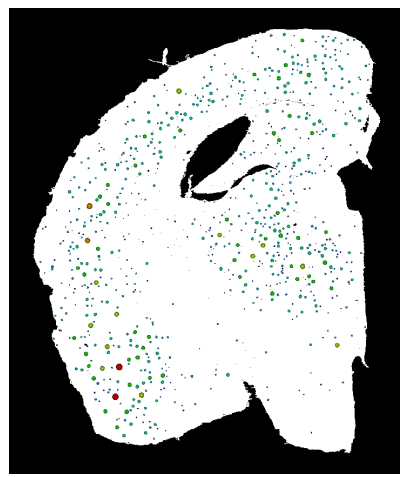

e)

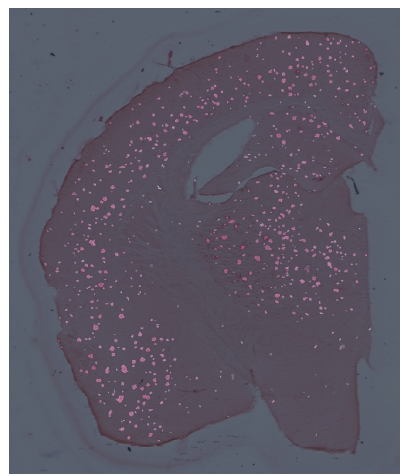

f)

Fig. 2. a) Coronal section stained using Congo Red, b) inverted and masked green color component, c) first and d) second seeds estimation and Voronoi computation. e) Final AP extracted with the automated method and f) image fusion of the original section with the detected AP (bright spots).

plaques were also superimposed to the original section to visually appreciate the reliability of the method (Fig 2 ).

In order to validate it, this methodology was applied to two selected brain regions from mice with large and small amyloid plaques ( $\mathrm{n}=5$ for each group). Amyloid load values were estimated in the targeted anatomical regions providing close intervals: $[0.75 ; 5.03]$ for the reference method and $[1.82 ; 5.63]$ for the automated method (Table 1). Expert and automated methods had a highly and significant correlation: $R=0.96, p<0.0001$.

The algorithm was implemented in $\mathrm{C}++$ and the computation was performed on a Linux Workstation (processor Intel Xeon 3.2GHz) with 2Go RAM and required two minutes to process a $1300 * 1300$ pixels image and half an hour for a brain constituted of 15 sections. A few hours were sufficient to process the 6 brains of the study. Curves representing plaques and brain tissue areas for each section according to the antero-posterior axis are presented in figure 3 a. 
Table 1. Results of amyloid load computed on hippocampus and frontal regions

\begin{tabular}{|c|l|l|l|l|l|l|l|l|l|l|}
\cline { 2 - 10 } \multicolumn{1}{c|}{} & 1 & 2 & 3 & 4 & 5 & 6 & 7 & 8 & 9 & 10 \\
\hline Frontal (expert) & 2.2 & 1.6 & 1.2 & 1.2 & 1.4 & 3.8 & 4.1 & 5.0 & 4.1 & 4.0 \\
\hline Frontal (method) & 2.7 & 2.1 & 1.9 & 2.1 & 2.0 & 5.6 & 4.5 & 5.4 & 4.6 & 5.3 \\
\hline Hippoc. (expert) & 1.6 & 0.9 & 1.3 & 0.8 & 1.4 & 3.6 & 3.5 & 4.8 & 4.3 & 4.4 \\
\hline Hippoc. (method) & 2.9 & 1.8 & 2.6 & 2.1 & 2.7 & 4.4 & 4.3 & 5.1 & 5.0 & 5.0 \\
\hline
\end{tabular}

Consistent values were obtained for the 6 brains for both brain tissue and AP classes. The increase of the areas observed for brain underlines the variability of section dimensions accross the whole brain. Moreover, AP constituted from $2.5 \%$ up to $6 \%$ of the global area of the section which corroborated the assumption in our modelling that tissue was the predominant class in the histological section. The histogram of figure $3 \mathrm{~b}$ displays the number of AP detected according to their areas for each brain. Despite the variation of the number of sections in the different brains $(12,13$ or 14 sections) the resulting information is characteristic and consistent with the literature and underlines the predominance of small AP over big AP in AD.
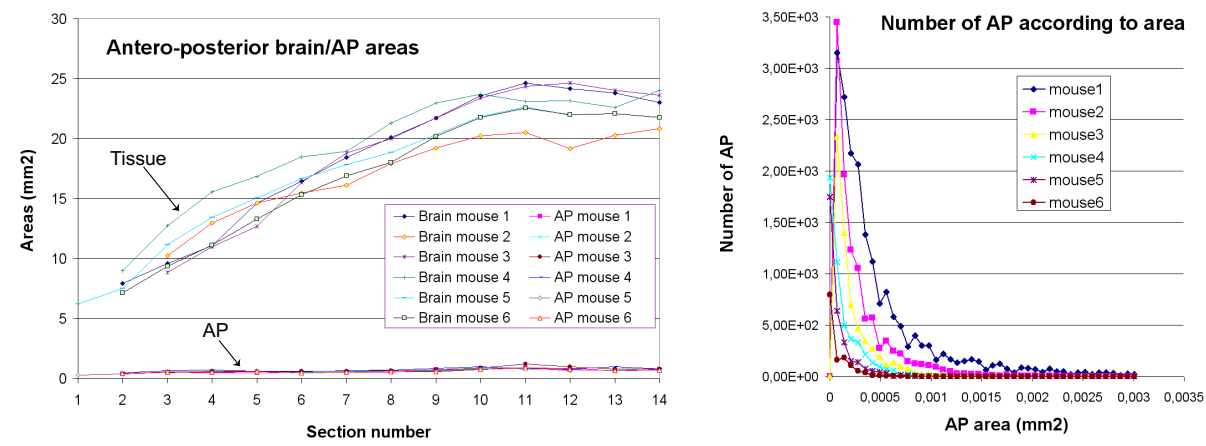

Fig. 3. a) Curves representing brain/AP areas calculated according to antero-posterior sections in 6 mouse brains. b) Histogram of the number of AP detected according to their area.

A 3-D surface rendering view representing AP as spheres for the sections of a single brain is presented in figure 4 to highlight the ability of the method to deal with 3 -D data. To facilitate the vizualization, inter-section space was artificially increased to $2 \mathrm{~mm}$. It is interesting to notice that AP were mainly located in the cortical areas but also in internal structures like thalamus and hippocampus (section 10, Fig 4). 


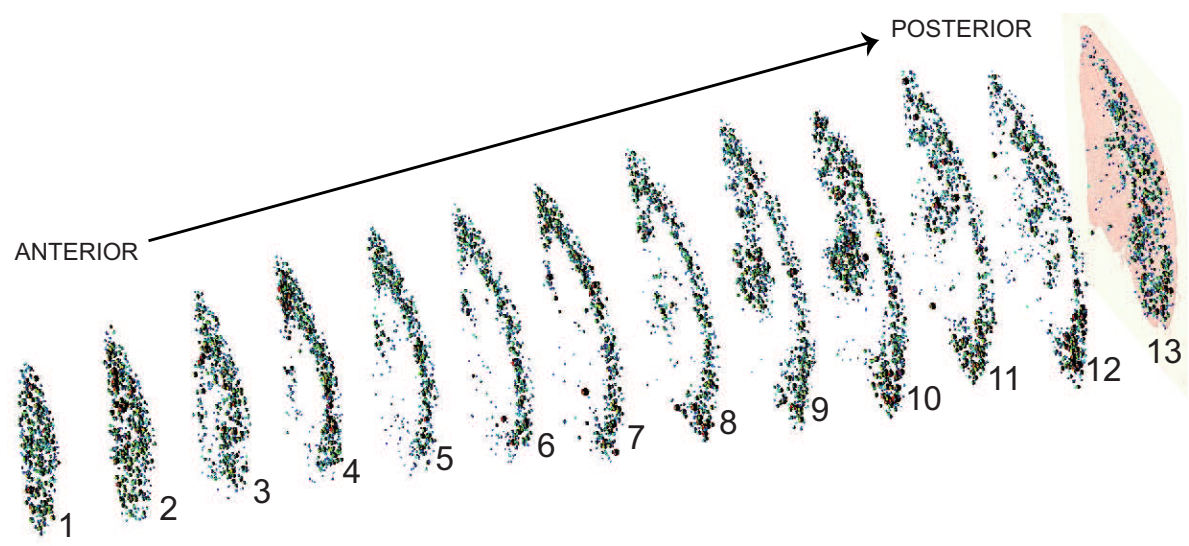

Fig. 4. 3-D surface rendering of the AP detected in a whole brain of transgenic mouse

\section{Discussion and Conclusion}

This paper presents an original method to detect AP in stained brain sections of Alzheimer transgenic mice. All the steps which classically require operator intervention have been automated (removal of the background, definition of the thresholds) and processing duration is compatible with biological studies. The adaptive extraction of the seeds and their corresponding Voronoi's partition allowed the detection of small plaques which are often missed with global threshold approaches. The oversegmentation of the seeds is well-adapted to the mathematical modelling we proposed because in one hand it provides a valuable initialization and on the other hand the estimation and classification processes are able to deal with false seeds detected as well as twin AP. A few cases of non-detection were visually noticed. They corresponded to major agregates in which individual AP could not be detected or to small Voronoi's cells in which the two classes expected were not present. Futher works can resolve these limitations but will probably not modify our preliminary results. The proposed model presents the advantages to be easy to implement, to converge rapidly (less than 10 iterations in average were necessary for each Voronoi's cell), and to present a high correlation with the reference segmentation performed by an expert. Superior values of amyloid load obtained by the method compared to the expert segmentation was observed in table 1 and can be attributed to the ability of our method to detect the diffuse region of the AP and to take into account partial volume effect which is not the case with the expert method (threshold based on entropy criterion). Moreover, the differences noticed between large/small amyloid plaques can be imputed to the major differences in the images (Fig.1c). The use of our methodoly could improve the reproducibility of the segmentation and allow to compare studies performed in different laboratories which constitutes a significative overhang. The field of appplication of our method is large and includes specific analyses on delineated anatomical region, brain sections or whole brain. Such a tool enables group studies and makes possible the extraction of 
a huge amount of information from large dataset improving their analysis and comparison. Perspectives of this work will be the use of anatomical atlases [8] to treat massively large dataset and the possibility to integrate complementary information acquired post mortem (autoradiography, histology) 910] or in vivo (MRI high resolution) 11] in 3 dimensions.

\section{Acknowledgements}

The two first authors contributed equally to this work. We would like to thank Nikos Paragios for his help, his advice and his availability, and Xavier Pennec for his medical imaging advices. The authors would also like to thank the Sanofi-Aventis neurodegenerative group for the generous gift of the animals involved in this study.

\section{References}

1. Cummings, B.J., Cotman, C.W.: Image analysis of beta-amyloid load in alzheimer's disease and relation to dementia severity. Lancet 346, 1524-1528 (1995)

2. Defigueiredo, R.J., Cummings, B.J., Mundkur, P.Y., Cotman, C.W.: Color image analysis in neuroanatomical research: application to senile plaque subtype quantification in alzheimer's disease. Neurobiol Aging 16, 211-223 (1995)

3. Chubb, C., Inagaki, Y., Sheu, P., Cummings, B., Wasserman, A., Head, E., Cotman, C.: Biovision: an application for the automated image analysis of histological sections. Neurobiol. Aging 27, 1462-1476 (2006)

4. Annese, J., Sforza, D.M., Dubach, M., Bowden, D., Toga, A.W.: Postmortem highresolution 3-dimensional imaging of the primate brain: blockface imaging of perfusion stained tissue. Neuroimage 30, 61-69 (2006)

5. Carson, C., Belongie, S., Greenspan, H., Malik, J.: Blobworld: Image segmentation using expectation-maximization and its application to image querying. IEEE PAMI 24, 1026-1038 (2002)

6. Duyckaerts, C., Godefroy, G.: Voronoi tessellation to study the numerical density and the spatial distribution of neurones. J. Chem. Neuroanat. 20, 83-92 (2000)

7. Prodanov, D., Nagelkerke, N., Marani, E.: Spatial clustering analysis in neuroanatomy: applications of different approaches to motor nerve fiber distribution. J. Neurosci. Methods 160, 93-108 (2007)

8. Chan, E., Kovacevic, N., Ho, S.K.Y., Henkelman, R.M., Henderson, J.T.: Development of a high resolution three-dimensional surgical atlas of the murine head for strains 129s1/svimj and c57bl/6j using magnetic resonance imaging and microcomputed tomography. Neuroscience 144, 604-615 (2007)

9. Ourselin, S., Roche, A., Subsol, G., Pennec, X., Ayache, N.: Reconstructing a 3d structure from serial histological sections. Image and Vision Computing 19, 25-31 (2001)

10. Dubois, A., Dauguet, J., Herard, A.S., Besret, L., Duchesnay, E., Frouin, V., Hantraye, P., Bonvento, G., Delzescaux, T.: Automated three-dimensional analysis of histologic and autoradiographic rat brain sections: application to an activation study. J. Cereb. Blood Flow Metab (in press, 2007)

11. Dhenain, M., Delatour, B., Walczak, C., Volk, A.: Passive staining: a novel ex vivo MRI protocol to detect amyloid deposits in mouse models of Alzheimer's disease. Magn. Reson. Med. 55, 687-693 (2006) 